\title{
A Distributed Generation based Residential Smart Gateway Grid
}

\author{
Logaranjith Kumar.K.N ${ }^{1}$, Dr.S.Thangavel Professor \& HOD ${ }^{2}$ \\ ${ }^{1}$ (Dept of EEE, K.S.Rangasamy College of Technology,India), \\ ${ }^{2}$ (Dept of EEE, K.S.Rangasamy College of Technology,India)
}

\begin{abstract}
Smart Grid (SG) is a modernization of electric system through the integration of new age information technologies, new strategic public policies and allows for the new uses of the electric grid, both in operations and through new customer side applications, that extract the benefits of more efficient operation, more efficient use of grid assets and more cost-effective expansion of the electric grid. A solar system is connected for the household purposes. The maximum power is obtained using MPPT algorithm as previously and for a satisfactory operation of whole system there is no control strategy was developed. But, here Neural Network $(N N)$ control scheme is used for satisfactory operation of the whole system. NN is used in the control scheme for MPPT gate drive and dc load priority. For this application MATLAB/ Simulink software is used. In a power supply system smart residential electricity is named as Smart Gateway Grid (SGG). SGG enable residential distributed generations and energy storage system to participate in system operation, which will improve the reliability of power supply and helps households secure a high quality service while reducing the cost of consumption. This paper focuses on the development of a renewable energy DG based residential power supply system. The output of PV is utilized first in the household purpose and the SGG realizes the desired power flow control in the grid-tied operation mode.
\end{abstract}

Keywords; DC load, distributed generation (DG), smart gateway grid, residential power supply system.

\section{INTRODUCTION}

Now days, a reliable with high quality electric supply is needed for the residential purpose due to the emergence of computers, sensitive loads and modern communications [1]. At present situation required an uninterrupted power for the air conditioning and medical equipments etc., are increased the demand of home power supply system due to which have to highly concentrate on the reliability of grid supplied power [2]. In recent years, Distributed Generation (DG) have more attention because of the ability to provide peak power, demand reduction, backup power, improved power quality and ancillary services of power grid [3]-[5].

On the other hand, now a day's more household electrical appliances are directly powered by DC power due to which conversion from the utility grid was needed [7]. But also energy efficient appliances operates in DC was increased to offers the potential to use DC directly and need to avoid the losses while converting to AC and back [8]. Practically AC power is used in all grid tied household and commercial building utilities. It is the time to construct a new electric power supply system for the household purpose or commercial buildings. SGG shows the development on the renewable energy DG based residential power supply system, which is an AC/DC hybrid residential distribution system. It is an intelligent home electrical energy supply and management system.

\section{CONCEPT OF SMARTWAY GRID}

Generally, a normal household electric power supply consists of three major parts: source, distribution and load. In which SGG plays the role of distribution side. A conceptual smart home energy system based on SGG is shown in Fig.1. In this system, here there are four major parts has been considered as follows: power source, distribution (SGG), load and energy storage unit.

Different types of power supplies are composed by the power source (e.g. solar power, utility grid, wind power and other DGs). The power distribution (SGG) delivers the power from sources to loads. This consists of the power electronic devices and power lines. The load is composed of different types of residential electrical appliances (e.g. Refrigerator, Induction stove, Air conditioner, Washing machine etc.,). Some of the residential electric appliances internally consume AC power and other consumes DC power. Normally batteries are used as the energy storage unit for the house hold purposes. This stores enough energy produced by the renewable energy DGs and /or to power the critical loads in an emergency. 


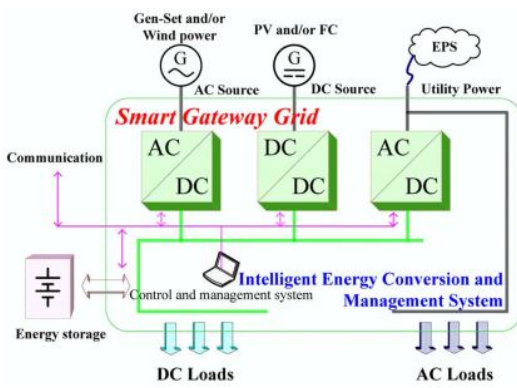

Fig. 1 Concept of SGG

SGG is the energy link between the power sources, loads and storages and makes them to work together. In SGG, not only AC bus and also DC bus is also available in order to meet the increasing of the DC loads. Even though the control of load according to the demand is not an new idea, the reason is that utilities play the key role and residential consumers as like the scheduled way of load management, which leads to attain while any discomfort of residential consumers the load will be disconnected.

Mainly the power control should be done to the needs of the residential consumers, purely not according to the utilities [10]. Therefore, an Intelligent Power and Energy Management System (IPMES) is required in the SGG. IPEMS is highly needed in order to monitor the whole system and also kindly adopt the power supply schedule to achieve maximum profit or the best service while meeting the constraints on demand, capacity and reliability [11]. In order to achieve the minimizing electricity payment and minimizing the waiting time for the operation of each residential appliances, the IPEMS should not treat the energy consumption scheduling problem as an optimization problem while the availability of real time electricity pricing [12]. It shows the IPEMS needs to automatically evaluate the priority of the appliances in real time since people depend on the different appliances at different time. Fig.2 shows the schematic of IPEMS.

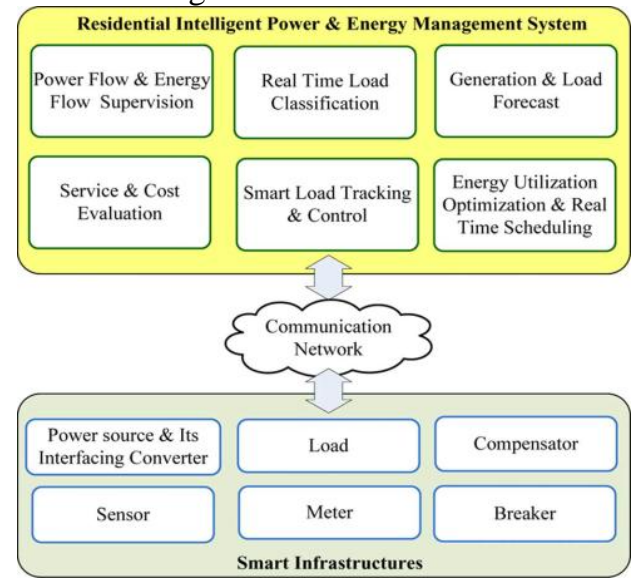

Fig.2 Schematic of IPEMS

The SGG provides modernized residential electric power supply with the following features:

1. The residential electric power system enhances the reliability by multiple power sources.

2. Feeding DC power directly to the residential electric appliances without any conversion, which improves the efficiency.

3. The excess energy from the renewable energy generations are feed to the storage and or / utility grid.

4. Adapting to customers response to the market and emergency.

5. Intelligent technique reduces the number of iterations in the MPPT technique.

6. Realizing long term profit with minimum environmental impact.

\section{SYSTEM CONFIGURATION OF SGG}

The SGG consists of a dc source connected converter (Module 1) and a grid-tied converter including an isolated dc-dc converter (Module 2) and a dc-ac inverter (Module 3). The isolated dc-dc converter can be connected to either the photovoltaic (PV) output port or $250-\mathrm{V}$ dc bus, which depends on which loads (ac loads or dc loads) are dominant in the system. Fig.3 shows a typical SGG principle structure which will be analyzed here.

In the system, the PV's $(1.1 \mathrm{~kW})$ main function is to provide energy to the dc loads $(1 \mathrm{~kW})$. If the load demand is lower than the PV output, the excess energy will be used to charge the battery. After the battery is fully charged, the energy can be converted from dc to ac in order to be used by ac loads ( $3 \mathrm{~kW})$ or fed into the utility grid. If the dc source is not available or is only partially available and the demand is on the dc loads, the 
battery will provide the energy. If the load demand is higher than the battery output, the ac grid will provide energy when it is available. The battery is designed to locally match the critical load demand for $1-4 \mathrm{~h}$.

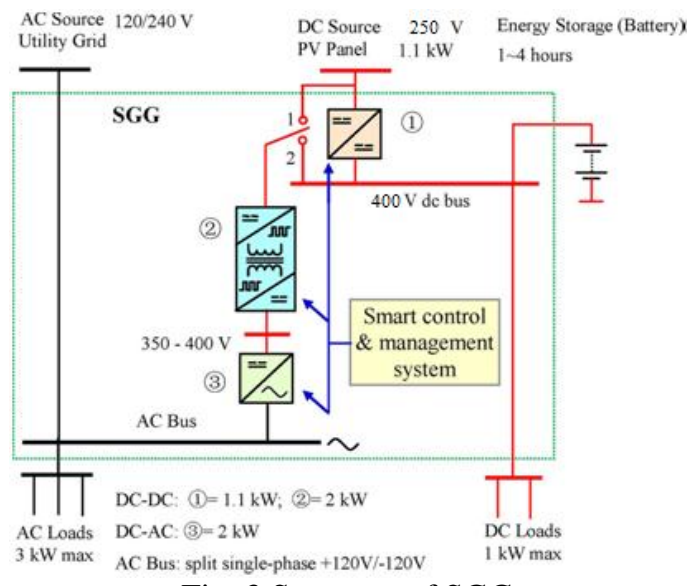

Fig. 3 Structure of SGG

Most dc loads in the home work under the voltage of $48-\mathrm{V}$ dc or below. This voltage level is also usually used for battery packs. Additionally, the voltage of $48-\mathrm{V}$ dc does not require any protection even against direct contacts. $250-\mathrm{V}$ is selected and used for the dc bus in the SGG to ensure safety. Certainly, the voltage level of the dc bus mainly depends on the load power and the length of the feeder. If the load is too heavy and the distribution line is too long, the high dc voltage will be required to avoid too large voltage drops and power losses.

As described above, the proposed configuration will meet the major requirements of the future residential building power supply system: high reliability coming from utilizing both the utility grid and the onsite PV and battery, high efficiency owing to directly feeding dc loads, and green because of integrated renewable energy DGs. More importantly, because multiple power sources and energy storage are integrated and power electronic converters are used popularly, both the consumer-dominating demand side management and pricing-responsive load management become possible, which is beneficial to achieve minimum cost of the electricity consumption for the residential users.

\section{TOPOLOGY SELECTION}

It is very important for residential applications to reduce the installation cost. So the low cost converter topologies are popular. Of course, these topologies should have high efficiency. As shown in Fig. 3, Module 1 is used to transmit electricity from the PV $(1.1 \mathrm{KW})$ to the dc bus $(500 \mathrm{~V})$. It is required to "buck" the PV voltage to meet the dc bus requirement. In addition, it should also perform the function of maximum power point tracking (MPPT) for the PV. Consequently, a basic buck converter is competent enough to perform the desired functions. Because only minimum switches are needed, Module 1 will have low cost and high efficiency. Module 2 and Module 3 are used to interconnect the dc bus and ac bus (the utility grid). The power flow through them is bidirectional. Because the utility grid is a single-phase 3-wire system with rated line-to-line voltage of $240 \mathrm{~V}$, the dc link voltage of the inverter should be rated at about 350-400 V. So a high step-up conversion ratio is required for Module 2. The use of a transformer can allow better converter optimization.

By proper choice of the transformer turns ratio, the voltage or current stress imposed on the switches and diodes can be minimized, leading to improved efficiency and lower cost. In addition, the transformer can provide galvanic isolation between the high voltage system and the low voltage system to satisfy the safety requirement. The detailed topology of Module 2 is bidirectional. A full-bridge converter is selected for the low voltage side (LVS) to achieve minimum voltage and current stresses on the devices. And an active voltage doubler is used for the high voltage side (HVS) to obtain a small transformer turns ratio and low current stress. For Module 2, the forward power flow is defined as from the LVS to the HVS unless specified otherwise.

Module 3 employs a three-leg PWM inverter to achieve the requirement of the split single-phase grid. It should be noticed that Module 3 shares a common two switch leg with Module 2. In this way, the function of voltage doubler and all the advantages of the six-switch inverter are achieved at the same time. Furthermore, the voltage doubler and inverter can be realized by using just one IPM in real circuit design. For Module 3, the forward power flow is defined as from the dc-link to ac side unless specified otherwise. From the analysis above, it can be seen that the proposed topology of the SGG, only has six MOSFETs for the low voltage dc stage (Module 1 and LVS of Module 2), one high frequency transformer, one IPM for the high voltage ac stage, and several filters. So it is low cost, reliable and easy to build, which makes it very potential for residential applications. 


\section{Operation Mode Of Sgg}

There are two main categories of different modes of operation, they are: Stand alone or Off-grid mode and Grid tied or Grid connected mode. By mainly considering these two categories there are eight operation modes for the Smart Gateway Grid has been done. Stand-alone operation is a very important function of the SGG to enhance the power supply reliability for the residential users. In this situation, PV is the only power generation, and the excess or deficient power is compensated by the battery. Because the outputs of the PV and the battery are limited, it is not permitted that all ac and dc loads are connected. Fortunately, most residential electric appliances are re-schedulable.

\subsection{Stand alone or Off-grid Mode}

In the stand-alone mode, the actual charging or discharging current of the battery depends passively on the PV supply and the on-site load demand since the battery directly connects to the dc bus. To protect the battery against an excessive charge, the PV should be disconnected from the SGG when the battery voltage increases above the maximum threshold and the current required by the loads is less than the current delivered by the PV. On the other hand, to protect the battery against excessive discharge, the loads (or converter) should be automatically shut down or disconnected when the battery voltage reaches the minimum threshold and the current required by the loads is bigger han that delivered by the PV. Obviously, above functions are realized in the IPEMS. Fig 4 shows stand alone mode.

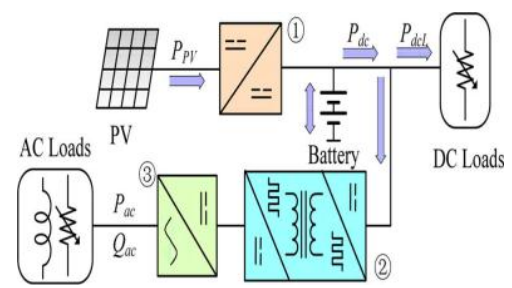

Fig. 4 First Category Operation Mode: Stand-alone

\subsection{Grid Tied or Grid Connected Mode}

The second category is the situation where the SGG is connected to the utility grid, as shown in Fig. 5, which is called "grid-tied" or "grid-connected" mode. In this situation, both the PV and the utility grid are power sources. The main function of the PV is to generate power and provide local power support. And the excess or deficient power of PV is compensated by the utility grid. If the PV output is more than the demand of the load and battery, the excess power will be transferred to the utility grid. Otherwise, the whole system will draw real power from the utility grid. With the interfacing converter (Module 3), controllable reactive power can also be produced by the SGG. As a result, the line loss can be reduced and the overall system efficiency can be improved.

Herein, the battery does not offset the intermittency of the PV output. It just acts as an emergency power supply to handle the unusual requirement. For example, if the electricity rate is extremely high and unacceptable, the power from the utility grid will be limited and the battery will be considered to provide power first.

Whether connecting the SGG to the utility grid really depends on the condition of the utility grid. If the utility grid is in normal operation, the SGG will be connected to it. Once there is a failure in the utility grid (e.g., short circuit fault), the SGG must be smoothly transferred to the stand-alone mode as soon as possible. For different operation modes, the three modules will work under different statuses which are outlined as follows.

a) Module 1: The direction of the power flow of Module 1 is always from the PV to the dc bus regardless of whether the SGG is in the stand-alone mode or not.

b) Module 2 and Module 3: The power can only flow through Module 2 and Module 3 from the dc bus to the ac bus in the stand-alone mode. However, in the grid-tied mode, the direction of the power flow of Module 2 and Module 3 will be from the dc bus to the ac bus if the PV output power is more than the dc load consumption, otherwise, the power flow direction will reverse. 


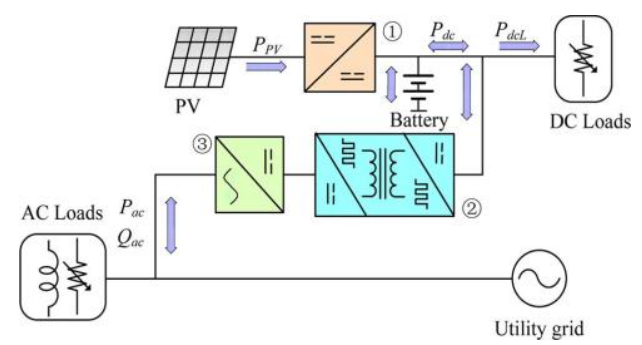

Fig.5 Second Category Operation Mode: Grid-Tied

In general, the operating states of the modules are determined by the power differences between the PV production plus battery output (Pdc) and dc load demand (PdcL) and ac side demand (Pac). If Pdc $>$ PdcL, the SGG will work on the forward grid-tied state (power flowing from the dc bus to the ac bus). And if Pdc $<$ PdcL, the SGG will work on the reverse grid-tied state (power flowing from the ac bus to the dc bus). It should be pointed out that the energy drawn from the utility grid will be limited if the electricity rate is too high. Fig.6 shows the transitions between different operation modes.

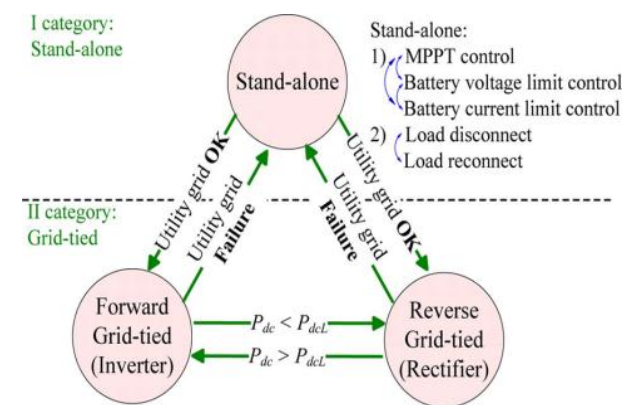

Fig. 6 Operation mode transition

\section{Module 1 Control}

When the buck converter works in the Continuous Conduction Mode (CCM), the input and output voltages are related as follows:

$$
V_{o d c}=D_{Q 1} \cdot V_{P V}
$$

where $\mathrm{DQ}_{1}$ is the duty cycle in the range of $0.5-1$.

When the converter operates in the Discontinuous Conduction Mode (DCM), the relation between the input and output voltages is (for the constant output voltage case).

$$
\begin{gathered}
V_{\text {odc }}=D_{Q 1} \cdot V_{P V} / \frac{\frac{I_{\text {odc }}}{I L B_{\max }}}{1-\frac{V_{\text {odc }}}{V_{V^{2}}}} \\
\text { Where, ILB } B_{\text {max }}=\frac{T_{S} V_{\text {odc }}}{2 L_{1}}
\end{gathered}
$$

Here, the duty cycle is the unique control variable to adjust the operating point of the buck converter. From (1), we can get

$$
d D_{Q 1} / d V_{P V}=-\mathrm{V}_{\text {odc }} / V_{P V}^{2}<0 \quad \text { (or) } \quad d V_{P V} / d D_{Q 1}=-\mathrm{V}_{\text {odc }} / D_{Q 1}^{2}<0
$$

We can also get the same conclusion from (2). Considering (3), and are in inverse proportion in both CCM and DCM. Thus, a reduction of duty cycle will cause an increment in output voltage of the PV array if the battery voltage keeps invariable. Therefore, a control scheme with the MPPT function can be designed for Module 1. An inner current loop is applied to limit the current.

Module 2 Control

Module 2 is a bidirectional voltage-fed dc-dc converter. Its primary and secondary voltages can be fully controlled. The phase shifting modulation method is proposed to reduce the switching loss by soft switching. The full-bridge converter and voltage doubler produce square voltages Vpri and Vsec. The power transferred through the transformer can be controlled by adjusting the phase shift between Vpri and Vsec as expressed by the following equation.

$$
P=V_{d c L}\left(V_{d c H} / 2 n\right)^{\varphi(\pi-\varphi)} /\left(2 \pi f_{s} L_{s} \pi\right)
$$

where $\varphi$ is the phase shift between LVS and HVS, fs is the switching frequency, and Ls is leakage inductance of the transformer. 
From (4), we can get

$$
d P / d \varphi=V_{d c L}\left(V_{d c H} / 2 n(\pi-2 \varphi) /\left(2 \pi f_{s} L_{s} \pi\right)\right)
$$

The phase shift varies in the range of $(0, \pi / 2)$ under forward power flow, thus

$$
d P / d \varphi>0
$$

Considering (6), an increase in phase shift will cause an increment in transferred power. The following equations can also be obtained from (4):

$$
\begin{aligned}
& \frac{d V_{d c L}}{d \varphi}=\frac{P 2 \pi f_{S} L_{S} \pi}{\left(V_{d c H} / 2 n\right)} \cdot \frac{2 \varphi-\pi}{\varphi^{2}(\pi-\varphi)^{2}} \\
& \frac{d V_{d c H}}{d \varphi}=\frac{P 2 \pi f_{S} L_{S} \pi}{\left(V_{d c L / 2 n}\right)} \cdot \frac{2 \varphi-\pi}{\varphi^{2}(\pi-\varphi)^{2}}
\end{aligned}
$$

As can be seen from (7) and (8), the voltages of LVS and HVS will decrease as the phase shift increases when it varies in the range of $(0, \pi / 2)$.

When the whole system works in the stand-alone mode, Module 2 must keep the HVS dc-link voltage constant. The voltage of the Cdc2 will increase if the input power (Pin) is larger than the output power (Pin) . This means the variation of the voltage of the $\mathrm{Cdc} 2$ indicates a power change or difference between Pin and Pout. So the voltage control loop can be used to substitute the power control loop.

Here, the dc-link voltage $d V_{d c H}$ is fed back to produce the phase shift command to control the power from the primary side to the secondary side. However, the isolated dc-dc converter may carry out the different control strategy in the grid-tied mode. Because the HVS dc-link voltage $d V_{d c H}$ can be controlled by Module 3 , which will be addressed later, Module 2 just needs to directly control the penetrating power by adjusting the phase shift.

Module 3 Control

In the stand-alone mode, the inverter has to control the frequency and voltage of the ac bus. It should keep the output ac voltage $\left(\mathrm{V}_{\mathrm{a}}\right.$ and $\left.\mathrm{V}_{\mathrm{b}}\right)$ constant. A control scheme of the inverter for the stand-alone mode consists of two control loops: the outer rms voltage loop and the inner instantaneous voltage loop. The Distributed Resources shall not actively regulate the voltage at the PCC (point of common coupling). So in the grid-tied mode, the inverter does not control the output ac voltages but control the output currents (or output power). At the same time, it is also expected to keep the dc-link voltage $\mathrm{V}_{\mathrm{dcH}}$ constant. A control scheme for the grid-tied mode consists of a dc-link voltage loop, an ac current loop, and an ac voltage feed forward loop. Surely, if the reactive power flow control is desired, the dc-link voltage loop can be used to produce the synchronous frame d-axis reference current and the reactive power control loop can be employed to produce the q-axis current.

\section{SIMULATION}

To verify the above design and analysis, a simulation model was developed in Matlab $\backslash$ Simulink, as shown in Fig. 7. In the simulation, both dc and ac loads are set at $500 \mathrm{~W}$, separately. The SGG is connected to the grid at $5 \mathrm{~s}$. Then, the maximum output power of PV varies from $1.1 \mathrm{~kW}$ to $1.01 \mathrm{~kW}$ at $5 \mathrm{~s}$, to $600 \mathrm{~W}$ at $0.3 \mathrm{~s}$, and back to $1.01 \mathrm{~kW}$ at $0.5 \mathrm{~s}$. Finally, at $1 \mathrm{~s}$, half of ac loads are switched off. The simulation results are shown in Fig. 8.

As can be seen, the SGG can always obtain the maximum output power from the PV during different time periods. From $0.1 \mathrm{~s}$ to $0.3 \mathrm{~s}$ and $0.5 \mathrm{~s}$ to $1.0 \mathrm{~s}$, because the output power of the PV is more than the demand of the dc bus, the excess power is transferred to the ac bus. At the same time, the utility grid also provides power to the ac loads. From $0.3 \mathrm{~s}$ to $0.5 \mathrm{~s}$, there is no real power transferred from the PV to the ac bus because the PV output is only sufficient for the dc demand. Only the grid powers the ac loads. After $1.0 \mathrm{~s}$, because the PV output is more than the dc and ac demand, the excess power is fed into the grid. 


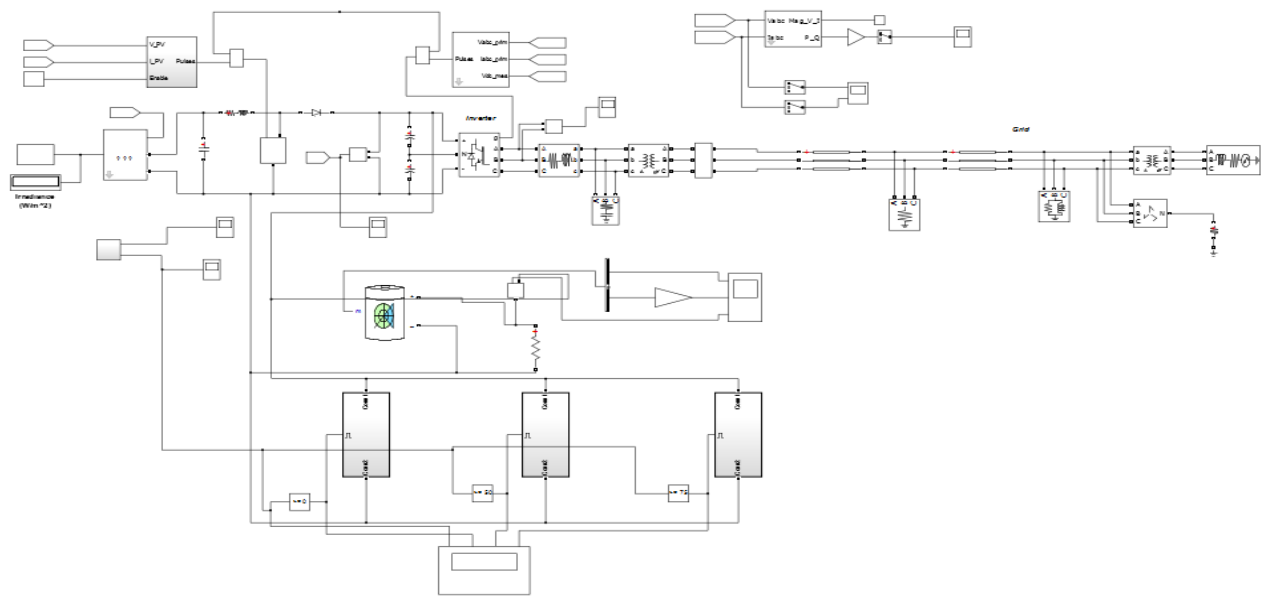

Fig 7 The Simulation Model in MatlablSimulink

The simulation results are shown in Fig.8. Fig.8(a) shows the Load 1 is connected, when the PV array provides the output power as greater than or equal to $0 \mathrm{KW}$. Load 2 is connected, when the PV array provides the output power as greater than or equal to $50 \mathrm{KW}$. Load 1 is connected, when the PV array provides the output power as greater than or equal to $75 \mathrm{KW}$. Fig.8 (b) shows the battery charging voltage, state of charging and discharging of battery and current.As a result, in the simulation, DC source connected converter realizes the MPPT for the PV, Grid tied converter including an isolated DC-DC converter and a DC-AC inverter realize the power flow control, and the operation mode transitions are automatically performed according to the power differences between the PV output and the demand. Therefore, the house can be powered more greenly, flexibly, and reliably.

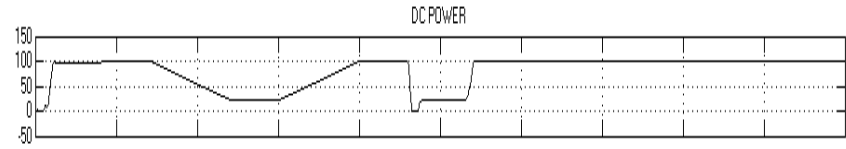

Lood 1 Enatle Signa
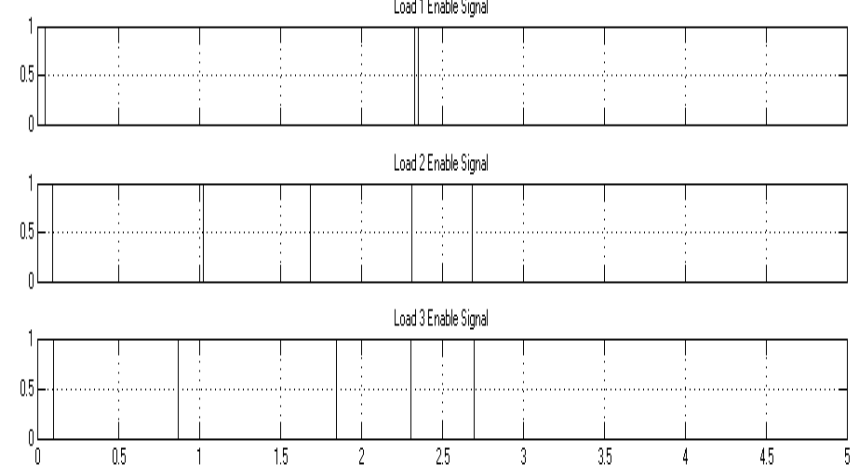

Fig. 8 (a) DC Power, Load Enable Signals.
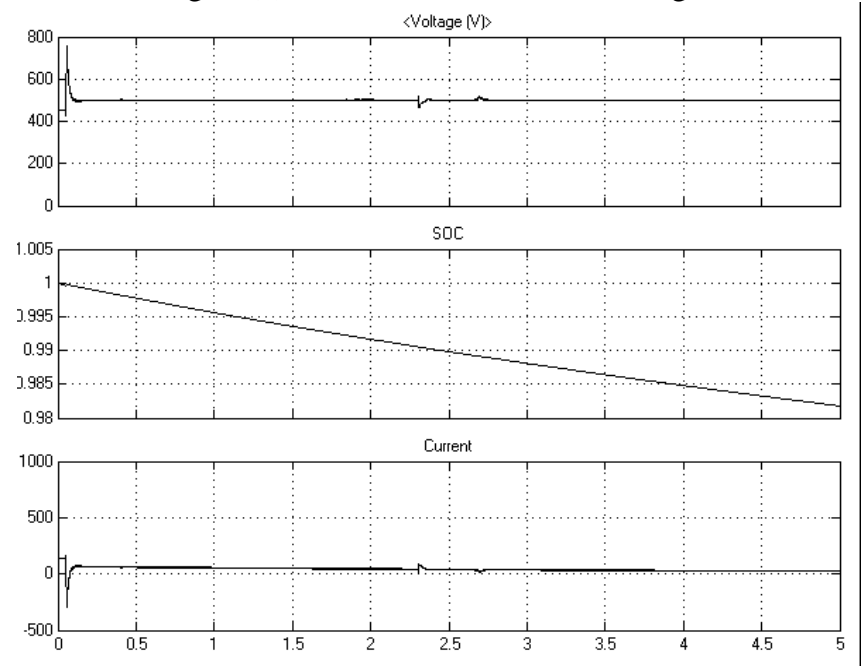

Fig. 8 (b) Battery Voltage, State of Charging and Current 


\section{CONCLUSION}

SGG can employ intelligent power and management system to monitor and control the entire system. It can integrate multiple power sources, such as the utility grid and various renewable energy DGs, and energy storage into the residential electric power supply system, which can improve the reliability of the power supply. Neural Network gives quick response, which reduces the number of iterations and error has been find quickly the MPPT drive is actuated. By implementing PI controller driven MPPT gate can obtain uninterrupted power supply. It obtains reliability of power supply and high quality services. The PV output is utilized first in the residential electric power supply system and the SGG realizes the desired power flow control in the grid-tied operation mode. Integrating DG and energy storage units makes the home energy supply more reliable, more efficient, and greener.The power electronic interfaces make the residential power supply system controllable and at the same time provide the approach to realizing intelligent home energy management. It will allow the customer to play an active role in the supply of electricity, which can help the utility grid respond to equipment failures, extreme weather conditions, etc.

Doubtless, an intelligent power and energy management system should be employed in the SGG to monitor and control the entire system. The SGG can be designed to be simple, efficient, and low cost, which makes it very promising for residential power supply. High reliability coming from utilizing both the utility grid and the on-site PV and battery, high efficiency owing to directly feeding dc loads, and green because of integrated renewable energy DGs. More importantly, because multiple power sources and energy storage are integrated and power electronic converters are used popularly, both the consumer-dominating demand side management and pricing-responsive load management become possible, which is beneficial to achieve minimum cost of the electricity consumption for the residential use.

\section{ACKNOWLEDGMENT}

A project of this nature needs co-operation and support from many persons for the successful completion. In this regard, I am fortunate to express my heartfelt thanks to the Head of the Department Dr. S. THANGAVEL, M.E., M.B.A., Ph.D., for his effective leadership, encouragement and guidance in the project.

\section{References}

[1] R. Strzelecki and G. Benysek, Power Electronics in Smart Electrical Energy Networks. London, U.K.: Springer, 2008.

[2] R. Zogg, T. Lawrence, K. Roth, and J. Brodrick, "Residential emergency power systems,” Ashrae J., vol. 48, pp. 68-70, Oct. 2006.

[3] W. Kramer, S. Chakraborty, B. Kroposki, and H. Thomas, "Advanced power electronic interfaces for distributed energy systems, part 1: Systems and topologies," National Renewable Energy Laboratory, Tech. Rep. No. NREL/TP-581-42672, Mar. 2008.

[4] H. Jiayi, J. Chuanwen, and X. Rong, "A review on distributed energy resources and MicroGrid," Renewable Sustainable Energy Rev., vol. 12, pp. 2472-2483, 2008.

[5] G. Pepermans, J. Driesen, D. Haeseldonckx, R. Belmans, and W. D'haeseleer, "Distributed generation: Definition, benefits and issues," Energy Policy, vol. 33, pp. 787-798, 2005.

[6] J. A. P. Lopes, N. Hatziargyriou, J. Mutale, P. Djapic, and N. Jenkins, "Integrating distributed generation into electric power systems: A re-view of drivers, challenges and opportunities,” Elec. Power Syst. Res., vol. 77, pp. 1189-1203, Jul. 2007.

[7] K. George, "DC power production, delivery and utilization," Electric Power Research Institute. Palo Alto, CA, Jun. 2006, White Paper.

[8] K. Garbesi, V. Vossos, A. Sanstad, and G. Burch, "Optimizing energy savings from direct-DC in U.S. residential buildings," Lawrence Berkeley National Laboratory, Berkeley, CA, Tech. Rep. LBNL-5193E, Oct. 2011.

[9] K. Engelen, E. L. Shun, P. Vermeyen, I. Pardon, R. D'Hulst, J. Driesen, andR. Belmans, "The feasibility of small-scale residential DC distribution systems," in Proc. 32ndAnnu. Conf. IEEE Ind. Electron. (IECON 2006), 2006, pp. 2618-2623.

[10] S. Tiptipakorn, W. J. Lee, C. Mao, and J. Lu, "Price naming on home appliance load controls in real-time pricing environment," in Proc. IEEE Power Energy Soc. Gen. Meet. 2010.

[11] D. Niyato, L. Xiao, and P. Wang, "Machine-to-machine communications for home energy management system in smart grid," IEEE Commun. Mag., vol. 49, pp. 53-59, Apr. 2011.

[12] H. Mohsenian-Rad and A. Leon-Garcia, "Optimal residential load control with price prediction in real-time electricity pricing environments," IEEE Trans. Smart Grid, vol. 1, pp. 120-133,2010.

[13] Sannino, G. Postiglione, and M. H. J. Bollen, "Feasibility of a DC network for commercial facilities," IEEE Trans. Ind. Appl., vol. 39, pp. 1499-1507, Sep.-Oct. 2003.

[14] R. W. Erickson and D. Maksimovic, Fundamentals of Power Electronics, 2nd ed. Norwell, MA: Kluwer Academic, 2001.

[15] N. Mohan, T. M. Undeland, and W. P. Robbins, Power Electronics: Converter, Applications and Design, 3rd ed. New York: Wiley, 2003.

[16] J. Wang, F. Z. Peng, J. Anderson, A. Joseph, and R. Buffenbarger, "Low cost fuel cell converter system for residential power generation," IEEE Trans. Power Electron., vol. 19, pp. 13 15-1322, Sep. 2004.

[17] F. Z. Peng, Y. W. Li, and L. M. Tolbert, "Control and protection of power electronics interfaced distributed generation systems in a customer-driven microgrid," in Proc. 2009 IEEE Power Energy Soc. Gen.Meet., vol. 1-8, pp. 1801- 1808, 5298. 
K.N.Logaranjith Kumar was born in Erode, Tamilnadu in 1987. He received the Bachelor degree in electrical engineering from Anna University, Chennai in 2009. He currently pursues his Master degree in power systems engineering at K.S.Rangasamy College of Technology, Tiruchengode. His research interests include uninterrupted residential electric power supply system and grid connection of alternative power supply by the concept of smart grid.

Dr S. Thangavel obtained his bachelor degree in Electrical and Electronics Engineering from Bharathiar University, India in 1993.He obtained his M.E., degree in Control and Instrumentation and Ph.D. for the thesis titled ' Intelligent controllers to Industrial Drives' from Anna University, Chennai in 2002 and 2008 respectively. He is working as Professor and Head in the Department of Electrical and Electronics Engineering, K.S.Rangasamy college of Technology, Tiruchengode, Tamilnadu, India. He had published papers in the area of Intelligent controllers for industrial drives in various International Journals and Conferences. His specific area of interest includes Intelligent controllers for Drives, Renewable Energy Sources. Dr. S.Thangavel is a member in ISTE, India and IEEE, USA. 